\title{
Endothelial Progenitor Cells and Endothelial Vesicles - What Is the Significance for Patients with Chronic Kidney Disease?
}

\author{
Rajesh Mohandas Mark S. Segal \\ Division of Nephrology, University of Florida, Gainesville, Fla., USA
}

\section{Key Words}

Endothelial progenitor cells · Bone-marrow-derived progenitor cells $\cdot$ Circulating endothelial cells $\cdot$ Endothelial microvesicles $\cdot$ Chronic kidney disease

\begin{abstract}
Endothelial progenitor cells are cells derived from the bone marrow that circulate in the bloodstream and can exhibit phenotypic characteristics of endothelial cells. They are thought to be involved in postnatal vasculogenesis and to potentially help repair injured endothelium. Circulating endothelial cells are mature endothelial cells in the circulation, and endothelial vesicles or microparticles are thought to be derived from the membranes of endothelial cells as a result of injury or activation. Recent research has focused on using these markers of endothelial injury and repair to assess the state of endothelial health. These efforts have been hampered by lack of uniformity in methodology and terminology. Recent developments in flow cytometry techniques have allowed better characterization and definition of these cells. We review the common techniques used to identify and isolate these cells, clinical studies in patients with chronic kidney disease (CKD) where they serve as markers of endothelial health and predictors of outcome, and possible mechanisms of progenitor cell dysfunction in CKD.
\end{abstract}

\section{KARGER}

Fax +4161306 1234

E-Mail karger@karger.ch

www.karger.com
(C) 2010 S. Karger AG, Basel

Accessible online at: www.karger.com/bpu
Chronic kidney disease (CKD) carries a high risk of mortality, most of which is cardiovascular in origin [1]. Traditional risk factors do not explain the unusual prevalence of atherosclerosis in this high-risk group [2]. Various etiologies like oxidative stress, inflammation and endothelial dysfunction are thought to contribute to the pathogenesis of atherosclerosis in patients with CKD. The 'response to injury hypothesis' sets the endothelium as a key mechanism for the development of atherosclerosis [3].

The view of the endothelium as an inert structure that merely served to line blood vessels was formally challenged by Furchgott and Zawadzki [4] as well as others. They demonstrated that vasodilatation in response to acetylcholine occurs only in the presence of an intact endothelium. We now know that this is largely the result of endothelium-derived nitric oxide and that the endothelium secretes several vasoactive substances including prostacyclins and endothelins. The endothelium also regulates the balance between prothrombotic and antithrombotic activities. In the quiescent state, the endothelium-derived nitric oxide and prostacyclin directly inhibit platelet aggregation and thrombomodulin inactivates thrombin. In response to low shear stress and other stressors, the endothelium becomes prothrombotic, secreting platelet-activating factor and expressing thromboplastin on cell membranes. The endothelium also plays 
a crucial role in initiating and maintaining inflammation. The endothelial cells express cell adhesion molecules which regulate the binding of inflammatory cells (monocytes and specific subsets of T lymphocytes) to the endothelium. Research in the past has focused on measuring these vasoactive substances, inflammatory markers or cell adhesion molecules to assess the state of the endothelium and quantify the risk of atherosclerosis. More recently investigators have begun to use the number and function of circulating cells to gain insights into the health of the endothelium. The focus has been on bone-marrow-derived progenitor cells playing a role in repairing the endothelium as well as circulating endothelial cells (CECs) and endothelial microparticles as markers of endothelial injury or activation.

\section{Defining and Characterizing Endothelial Progenitor Cells}

In 1997, Asahara et al. [5] first demonstrated the existence of cells in the circulation that can differentiate into endothelial cells. This aroused considerable interest because of the potential for so-called endothelial progenitor cells to serve as markers of an individual's capacity to carry out repair of injured endothelium as well as the ability to use these cells in a therapeutic manner. Since then a large body of literature has accumulated concerning abnormal endothelial progenitor cell number and function in various disease states. However, lack of uniformity in terminology and methodology has caused considerable confusion in characterizing endothelial progenitor cells. Moreover what is termed an endothelial progenitor cell might not be a homogeneous population but derived from or requiring interaction of different cell sources including bone marrow, peripheral blood and even the vessel wall.

The two common methods to characterize endothelial progenitor cells are cell culture and flow cytometry. Depending on cell culture characteristics, endothelial progenitor cells have been characterized as early and late. Early progenitor cells or CFU-Hill colonies, as first described by Hill et al. [6], have been widely used in part because they have been validated as a marker for overall endothelial health and because of readily available commercial kits. These cells form colonies within a week when peripheral blood mononuclear cells are grown in angiogenic media. However, these cells have limited proliferative and angiogenic potential, and Rohde et al. [7] demonstrated that the central core of these colonies are inflammatory T cells. Sieveking et al. [8] used transwell cultures to demonstrate that the angiogenic effects of these cells may be due to paracrine effects. Yoder et al. [9] used clonal analysis to establish that the CFU-Hill colonies are of hematopoietic origin and express the common leukocyte antigen CD45 as well as the monocyte antigen CD14. Thus, these cells might be more appropriately termed 'bone-marrow-derived progenitor cells' (BMDPCs) and may be required for endothelial angiogenesis but may not directly give rise to endothelial cells. However, it is important to remember that while these cells may not be 'true endothelial progenitor cells' they may play a role in angiogenesis, and the number and function of these cells have been shown to correlate inversely with cardiovascular risk in a number of studies $[6,10]$.

In contrast late or outgrowth endothelial progenitor cells or endothelial outgrowth cells appear after 2-3 weeks of culture, and have enhanced proliferative and vasculogenic potential. They lack the monocyte marker CD45 but express the endothelial marker CD34. Sieveking et al. [8] used a tubulogenesis assay to demonstrate that these cells independently formed tubules and incorporate into differentiated endothelial cell tubules. These cells have been shown to be mobilized after myocardial infarction [11], can attenuate intimal hyperplasia after vascular injury and can form blood vessels in vivo in mouse models $[9,12]$. These colonies are rare, methods to isolate them cumbersome, and studies linking them to cardiovascular outcomes are still lacking.

Flow cytometry, as a method of enumerating BMDPCs, has the advantage of being sensitive, reproducible, relatively easy to perform and is the method of choice to directly detect circulating progenitor cells. However, there are limitations with regard to BMDPCs - the relative rarity of these cells and the difficulty in characterizing them simply on the basis of surface antigens. Moreover these undifferentiated cells may lose or acquire other surface antigens as they mature. BMDPCs are generally characterized by markers of immaturity as well as markers of endothelial lineage. The commonly used markers of immaturity are CD34 and/or CD133. CD34 is a transmembrane sialomucin protein that is expressed on early hematopoietic cells. However, it is nonspecific and is expressed on megakaryocytes and several tumor cells. It is an important adhesion molecule, though its exact function remains largely unknown. CD133 is a pentaspan transmembrane glycoprotein selectively expressed on hematopoietic and progenitor stem cells. It binds cholesterol, though its exact function remains unknown as well. 
The usual endothelial markers used are KDR/Flk-1 or vascular endothelial growth factor receptor 2, von Willebrand factor, vascular endothelial cadherin or CD146. Various groups have characterized endothelial progenitor cells as CD34+ KDR+, CD34+CD133+ and KDR+, or CD45-CD34+ as well as other combinations of these markers. Each of these has its own advantages and disadvantages. Since Asahara et al. [5] used CD34+KDR+ to identify putative endothelial progenitor cells, these markers have been used to define BMDPCs by flow cytometry. Though they have the advantage of being the only subset which has been correlated with cardiac outcomes in patients with CKD [13], we and others have found that commercially available KDR antibodies are plagued by reliability issues. Case et al. [14] included a marker of immaturity as well to define a BMDPC (CD34+KDR+CD133+) and to separate them from mature endothelial cells. However, recent studies have suggested that these cells as well may be hematopoietic precursor cells and have limited angiogenic potential. Recently several authors have used polychromatic flow cytometry to better characterize these circulating cells [15]. They identified BMDPCs as $\mathrm{CD} 31+\mathrm{CD} 34^{\text {bright }} \mathrm{CD} 45^{\mathrm{dim}} \mathrm{CD} 133+$ cells and CECs as CD$31^{\text {bright }} \mathrm{CD} 34^{\mathrm{dim}} \mathrm{CD} 45-\mathrm{CD} 133$ - cells. These techniques seem promising but further studies are needed to characterize the size and morphology of these cells and correlate with culture and cell isolation methods.

Flow cytometry, though useful to characterize circulating endothelial progenitor cells, does not lend itself to the rapid and efficient isolation of viable progenitor cells. To isolate these cells, protocols have utilized magnetic beads conjugated with specific antibodies against surface markers. Selecting for CD34 enriches for progenitor cells that promote angiogenesis. However, this population is inhomogeneous and includes other cells including angiogenic monocyte and macrophage progenitors.

\section{BMDPCs as Markers of Endothelial Health and Predictors of Outcomes in CKD}

CKD is characterized by reduced numbers of circulating BMDPCs as enumerated by flow cytometry, as well as decreased colony formation. Patients with CKD also show abnormal progenitor cell function including migration, adhesion and incorporation. Migration is assessed by the ability of these cells to migrate towards a potent chemotactic agent like SDF-1, adhesion by the ability to form a monolayer with mature endothelial cells and incorporation by a tube formation assay. From a vascular risk viewpoint, the functional characteristics of progenitor cells might be even more significant than the absolute numbers of such cells. These defects in progenitor cell function seem to start early in the disease. Krenning et al. [16] studied 50 patients with varying degrees of CKD and found that even stage I CKD was associated with a reduced number of circulating CD34+ cells and abnormal function and this worsened with more advanced renal failure.

Most studies in patients on hemodialysis have similarly found a reduced number and function of progenitor cells $[17,18]$. Although one study did show increased progenitor cell numbers, in this study the patients were carefully selected to exclude those with diabetes and vascular disease and thus they were not representative of the typical dialysis population [19]. Even though end-stage kidney disease is associated with low BMDPC numbers and dysfunction, in patients who have been longitudinally followed, institution of dialysis seems to partially improve BMDPC number and function [20]. This is also corroborated by studies demonstrating that BMDPC numbers correlate with dialysis efficacy, as measured by $\mathrm{Kt} / \mathrm{V}$ [21], and BMDPC function is improved with longer dialysis sessions such as in nocturnal hemodialysis [22]. Patients on peritoneal dialysis show similar results to those on hemodialysis, although in the one study that examined peritoneal dialysis and hemodialysis patients, the decrease in progenitor cell number was more dramatic in patients on hemodialysis [23].

Though there are several studies on BMDPC number and function in end-stage kidney disease, there are relatively few looking at outcomes. Schmidt-Lucke et al. [13] followed 77 patients on hemodialysis for a period of 10 months and found that reduced CD34+KDR+ cells conferred a hazard ratio of 3.9 for vascular events even after adjusting for all traditional risk factors. Nontraditional risk factors like coronary calcification do not seem to correlate with BMDPC number either [17]. Thus, CKD may be an independent risk factor for progenitor cell dysfunction, and this is at least in part ameliorated by renal replacement therapy and/or transplantation [24].

\section{Mechanisms of BMDPC Dysfunction in CKD}

While it is evident that progenitor cell function is impaired in $\mathrm{CKD}$, the exact mechanisms for this dysfunction are unclear. Reduced BMDPC number could be due to defective mobilization from the bone marrow, shortened survival or defective proliferation. Endothelial ni- 
tric oxide synthase is essential to mobilize BMDPCs from the bone marrow $[25,26]$. However, CKD is associated with elevated levels of asymmetric dimethylarginine, an endogenous inhibitor of endothelial nitric oxide synthase. In addition, diabetes, a condition prevalent in CKD patients, is associated with a deficiency of nitric oxide derived from endothelial nitric oxide synthase. We have previously shown that migration defects in CD34+ cells is due to cytoskeletal alterations which can be corrected by exogenous administration of nitric oxide [27].

Another potential mechanism of BMDPC dysfunction is inflammation. Various inflammatory markers like interferon, interleukin 6 or C-reactive protein have been shown to affect BMDPC function [28, 29]. Inflammation might act in part by causing BMDPC senescence and apoptosis. Several agents including angiotensin II and oxidized low-density lipoprotein have been shown to induce apoptosis of BMDPC through oxidative stress induction and reduction of telomerase activity $[30,31]$. Uremia or some of the uremic toxins like homocysteine [32] or $p$-cresol [33] may inhibit BMDPC differentiation and function. Lastly, complications of CKD such as lack of erythropoietin or secondary hyperparathyroidism may also contribute to BMDPC dysfunction.

\section{CECs and Microvesicles}

Though CECs were first reported almost 30 years ago, they have only been widely studied since the development of endothelial cell-specific monoclonal antibodies. These mature CECs are derived from the vessel wall and exhibit phenotypic endothelial markers such as von Willebrand factor, vascular endothelial cadherin or CD146. Though not clearly elucidated, potential mechanisms for the presence of these cells in the circulation include apoptosis and mechanical disruption. Several authors have reported increased numbers of CECs in response to a variety of stresses or pathological conditions. We have demonstrated that the CECs are elevated in subjects with hypertension, diabetes and on hemodialysis [34]. In a long-term follow-up study of 29 hemodialysis patients, we found that patients with elevated CECs had a 7-fold risk of vascular events compared to those with low levels of CECs [35]. The number of CECs showed no correlation with markers of inflammation or endothelial dysfunction. It has been suggested that the balance between CECs (which are indicative of endothelial injury) and BMDPCs (which can potentially repair damaged endothelium) might be more indicative of overall health of the vascular endothelium [36].

Endothelial microvesicles or microparticles are vesicles formed by the endothelial cell membrane after injury or activation. They lack a viable nucleus but express endothelial surface markers and/or cytoplasmic elements. These microparticles may also serve as a marker of endothelial injury and are elevated in numerous pathological states including hypertension, diabetes, coronary artery disease and end-stage renal disease. Circulating levels of CD144+ microparticles were found to correlate independently and inversely with flow-mediated brachial artery vasodilatation in patients with end-stage renal disease [37]. Though there are no outcome studies in kidney disease, in patients with coronary artery disease the number of annexin $\mathrm{V}+$ particles has been shown to be an excellent predictor of myocardial infarction and death [38]. There has been some controversy regarding whether these microparticles are just the sequela of vascular injury or have a pathological role. Several in vitro studies have shown that microparticles have proinflammatory as well as procoagulant effects and impair the release of nitric oxide from endothelial cells $[39,40]$. On the other hand, they also stimulate angiogenesis and differentiation of progenitor cells [41]. It is not certain to what extent these apparently counteracting mechanisms are involved in vivo, and certainly more research is needed in this area.

The recent application of new techniques has brought better understanding and characterization of BMDPCs, CECs and microparticles. Their role as markers of endothelial health in kidney disease and as predictors of survival and vascular events has been begun to be validated. However, their mechanistic role in postnatal vasculogenesis is still uncertain. Hopefully, the new knowledge gained will serve to effectively use these circulating cells as a predictor of health and disease and potentially as a therapeutic tool.

\section{Acknowledgements}

This work was supported by NIH EY012601-08A1, R01 HL091005-02, the Gatorade Research Funds and an ALR grant to M.S.S., a Genzyme Fellowship grant and NIH T32-DK07518 to R.M. 


\section{References}

$>1$ Go AS, et al: Chronic kidney disease and the 15 Duda DG, et al: A protocol for phenotypic risks of death, cardiovascular events, and hospitalization. N Engl J Med 2004;351: 1296-1305.

$>2$ Weiner DE, et al: The Framingham predictive instrument in chronic kidney disease. J Am Coll Cardiol 2007;50:217-224.

$\checkmark 3$ Ross R: Atherosclerosis - an inflammatory disease. N Engl J Med 1999;340:115-126.

4 Furchgott RF, Zawadzki JV: The obligatory role of endothelial cells in the relaxation of arterial smooth muscle by acetylcholine. $\mathrm{Na}-$ ture 1980;288:373-376.

5 Asahara T, et al: Isolation of putative progenitor endothelial cells for angiogenesis. Science 1997;275:964-967.

$\checkmark 6$ Hill JM, et al: Circulating endothelial progenitor cells, vascular function, and cardiovascular risk. N Engl J Med 2003;348:593600.

$\checkmark 7$ Rohde E, et al: Immune cells mimic the morphology of endothelial progenitor colonies in vitro. Stem Cells 2007;25:1746-1752.

$>8$ Sieveking DP, et al: Strikingly different angiogenic properties of endothelial progenitor cell subpopulations: insights from a novel human angiogenesis assay. J Am Coll Cardiol 2008;51:660-668.

$\checkmark 9$ Yoder MC, et al: Redefining endothelial progenitor cells via clonal analysis and hematopoietic stem/progenitor cell principles. Blood 2007;109:1801-1809.

10 Michowitz Y, et al: Circulating endothelial progenitor cells and clinical outcome in patients with congestive heart failure. Heart 2007;93:1046-1050.

-11 Massa M, et al: Rapid and large increase of the frequency of circulating endothelial colony-forming cells (ECFCs) generating late outgrowth endothelial cells in patients with acute myocardial infarction. Exp Hematol 2009;37:8-9.

$>12$ Wang C-H, et al: Late-outgrowth endothelial cells attenuate intimal hyperplasia contributed by mesenchymal stem cells after vascular injury. Arterioscler Thromb Vasc Biol 2008;28:54-60.

13 Schmidt-Lucke C, et al: Reduced number of circulating endothelial progenitor cells predicts future cardiovascular events: proof of concept for the clinical importance of endogenous vascular repair. Circulation 2005; 111:2981-2987.

14 Case J, et al: Human CD34+AC133+VEGFR$2+$ cells are not endothelial progenitor cells but distinct, primitive hematopoietic progenitors. Exp Hematol 2007;35:1109-1118. detection and enumeration of circulating endothelial cells and circulating progenitor cells in human blood. Nat Protoc 2007;2: 805-810.

16 Krenning G, et al: Endothelial progenitor cell dysfunction in patients with progressive chronic kidney disease. Am J Physiol Renal Physiol 2009;296:F1314-F1322.

17 Schlieper G, et al: Predictors of low circulating endothelial progenitor cell numbers in haemodialysis patients. Nephrol Dial Transplant 2008;23:2611-2618.

18 Eizawa T, et al: Circulating endothelial progenitor cells are reduced in hemodialysis patients. Curr Med Res Opin 2003;19:627633.

19 Herbrig K, et al: Increased total number but impaired migratory activity and adhesion of endothelial progenitor cells in patients on long-term hemodialysis. Am J Kidney Dis 2004;44:840-849.

20 de Groot K, et al: Uremia causes endothelial progenitor cell deficiency. Kidney Int 2004; 66:641-646.

21 Choi J-H, et al: Decreased number and impaired angiogenic function of endothelial progenitor cells in patients with chronic re nal failure. Arterioscler Thromb Vasc Biol 2004;24:1246-1252.

22 Chan CT, Li SH, Verma S: Nocturnal hemodialysis is associated with restoration of impaired endothelial progenitor cell biology in end-stage renal disease. Am J Physiol Renal Physiol 2005;289:F679-F684.

23 Ueno H, et al: Dialysis modality is independently associated with circulating endothelial progenitor cells in end-stage renal diseases patients. Nephrol Dial Transplant 2009, E-pub ahead of print.

24 Steiner S, et al: Endothelial progenitor cells in kidney transplant recipients. Transplantation 2006;81:599-606.

25 Aicher A, et al: Essential role of endothelial nitric oxide synthase for mobilization of stem and progenitor cells. Nat Med 2003;9: 1370-1376.

26 Gallagher KA, et al: Diabetic impairments in NO-mediated endothelial progenitor cell mobilization and homing are reversed by hyperoxia and SDF-1 alpha. J Clin Invest 2007; 117:1249-1259.

27 Segal MS, et al: Nitric oxide cytoskeletal-induced alterations reverse the endothelial progenitor cell migratory defect associated with diabetes. Diabetes 2006;55:102-109.

28 Fujii H, et al: C-reactive protein alters antioxidant defenses and promotes apoptosis in endothelial progenitor cells. Arterioscler Thromb Vasc Biol 2006;26:2476-2482.
29 Verma S, et al: C-reactive protein attenuates endothelial progenitor cell survival, differentiation, and function - further evidence of a mechanistic link between C-reactive protein and cardiovascular disease. Circulation 2004;109:2058-2067.

30 Imanishi T, et al: Oxidized low-density lipoprotein induces endothelial progenitor cell senescence, leading to cellular dysfunction. Clin Exp Pharmacol Physiol 2004;31:407413.

31 Imanishi T, Hano T, Nishio I: Angiotensin II accelerates endothelial progenitor cell senescence through induction of oxidative stress. J Hypertens 2005;23:97-104.

32 Chen JZ, et al: Effects of homocysteine on number and activity of endothelial progenitor cells from peripheral blood. J Mol Cell Cardiol 2004;36:233-239.

33 Dou L, et al: The uremic solutes $p$-cresol and indoxyl sulfate inhibit endothelial proliferation and wound repair. Kidney Int 2004;65: 442-451.

34 Koç M, Bihorac A, Segal MS: Circulating endothelial cells as potential markers of the state of the endothelium in hemodialysis patients. Am J Kidney Dis 2003;42:704-712.

-35 Koc M, et al: Circulating endothelial cells are associated with future vascular events in hemodialysis patients. Kidney Int 2005;67: 1078-1083.

36 Rodríguez-Ayala E, et al: Imbalance between detached circulating endothelial cells and endothelial progenitor cells in chronic kidney disease. Blood Purif 2006;24:196-202.

37 Brodsky SV, et al: Endothelium-derived microparticles impair endothelial function in vitro. Am J Physiol Heart Circ Physiol 2004; 286:H1910-1915.

38 Mallat Z, et al: Elevated levels of shed membrane microparticles with procoagulant potential in the peripheral circulating blood of patients with acute coronary syndromes. Circulation 2000;101:841-843.

39 Amabile N, et al: Circulating endothelial microparticles are associated with vascular dysfunction in patients with end-stage renal failure. J Am Soc Nephrol 2005;16:33813388.

40 Boulanger CM, et al: Circulating microparticles from patients with myocardial infarction cause endothelial dysfunction. Circulation 2001;104:2649-2652.

41 Hristov M, et al: Apoptotic bodies from endothelial cells enhance the number and initiate the differentiation of human endothelial progenitor cells in vitro. Blood 2004;104: 2761-2766. 